\title{
Application of Virtual Reality for Helping People with Psychiatric Disorders: A Mini Review
}

\author{
Mahdiyeh Sarraf-Razavi ${ }^{1}$, Seyyed Mohammad Tabatabaei ${ }^{1,2,3 *}$, Ali Talaei ${ }^{4}$, \\ Mohammad Reza Fayyazi Bordbar ${ }^{4}$ \\ ${ }^{1}$ Division of Neurocognitive Sciences, Psychiatry and Behavioral Sciences Research Center, \\ Mashhad University of Medical Sciences, Mashhad, Iran. \\ ${ }^{2}$ Medical Informatics Department, School of Medicine, Mashhad University of Medical \\ Sciences, Mashhad, Iran \\ ${ }^{3}$ Clinical Research Unit, Mashhad University of Medical Sciences, Mashhad, Iran \\ ${ }^{4}$ Psychiatry and Behavioral Sciences Research Center, Mashhad University of Medical Sciences, \\ Mashhad, Iran.
}

ISSN: 2576-8816

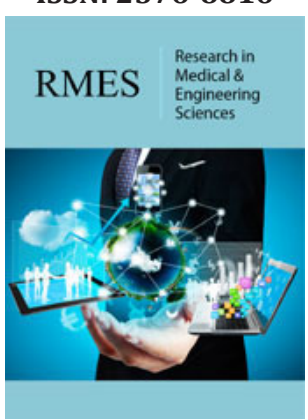

*Corresponding author: Seyyed Mohammad Tabatabaei, Ibn-e-Sina Street, Imam Reza Hospital, Department of Central Library, Clinical Research Unit, Mashhad, Iran

\section{Submission: 淟 August 05, 2019}

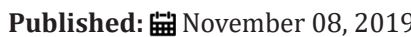

Volume 8 - Issue 2

How to cite this article: Seyyed Mohammad Tabatabaei, Mahdiyeh Sarraf Razavi, Ali Talaei and Mohammad Reza Fayyazi Bordbar. Application of Virtual Reality for Helping People with Psychiatric Disorders: A Mini Review. Res Med Eng Sci. 8(2).RMES.000684.2019.

DOI: 10.31031/RMES.2019.08.000684

Copyright@ Seyyed Mohammad Tabatabaei, This article is distributed under the terms of the Creative Commons Attribution 4.0 International License, which permits unrestricted use and redistribution provided that the original author and source are credited.

\begin{abstract}
Psychiatric disorders are characterized by behavioral and cognitive deficits. It is crucial to develop intervention strategies helping individuals with psychiatric disorders, their caregivers and educators in daily life. To this purpose, eXtended Reality (XR), i.e. a simulation of the real world based on computer graphics, can be useful as it allows instructors and therapists to offer a safe, repeatable and diversifiable environment during learning. This mini review examines studies that have investigated the use of XR in psychiatric disorders. Virtual reality is a promising intervention with several potential applications about behavioral and cognitive rehabilitation treatment in psychiatric disorders.
\end{abstract}

Keywords: Psychiatric disorders; Virtual reality; Cognitive rehabilitation

\section{Introduction}

Technology has been developing rapidly, especially in the area of Information and Communication. The development encompasses all sciences including heath and it introduces a new field called "electronic health". Electronic health, as a modern development, can promote social health, resulting in greater work efficiency, better quality of services and the return of investments [1]. One of the most recent technologies in the area of Electronic Health is the combination of real world with digital world. There are different kinds of combination including augmented reality (AR), virtual reality (VR), and mixed reality (MR) [2]. Actually, MR is the mixture of both the real and virtual, where the virtual augments the real or so on the contrary [3]. While AR combines virtual and real objects simultaneously, VR make the users able to control and navigate their movements in a stimulated real or unreal world $[4,5]$. As these technologies improved, it seemed they should be integrated and described as parts of a single concept. So, a new term, Extended Reality (XR), was born. XR is defined as the combination of all real and virtual environments with human machine interactions generated by computer technology using wearable devices [6]. Actually, it creates the illusion to make us feel as we are in a completely novel and different digital world [7]. Nowadays, many researchers and consumers have access to XR hardware and software. This technology has been caused major changes in research and practice in the field of psychology, makes us capable of deeply understanding human behavior and to be able to roll out training or therapies to everyone [8]. XR-based interventions on patients with mental or physical dysfunctions has been studying since the late $90 \mathrm{~s}$. the results of these studies show that this technology improves the quality of physical and mental health care while reducing its cost, so it should be considered as a viable and valuable option to for the treatment of mental or even physical conditions which may lead to disability [9]. So, we highlight studies evaluating the utility and efficacy of XR based treatments for psychiatric disorders.

\section{Methods}

We reviewed XR based studies in the area of psychiatric disorders published over the last 9 years. We used PsycINFO, PubMed, and Medline electronic databases to identify studies from 2010 to 2019, based on the following keywords: psychiatric disorders, extended 
reality, augmented reality, virtual reality, mixed reality, cognitive rehabilitation and therapy.

\section{Results}

According to the most of studies, XR usage can be categorized into 5 area based on the disorders. These 5 categories are: schizophrenia, autism spectrum disorders, attention deficit hyperactivity disorders, mood disorders and anxiety disorders which will be discussed in the following:

\section{Schizophrenia}

There were above 20 studies about using XR for schizophrenia and related problems, concerning assessment, assessing paranoia, treatment [10], improving social dysfunction. negative symptoms, psychopathology, social anxiety and discomfort, avoidance and social functioning, improve social cognition and performance of patients as well as generalization of the learned responses into patient's daily life [11], improve cognitive functioning in people with schizophrenia in WCST, WAIS-III sub-tests, Strop Test, and The Subjective Scale to Investigate Cognition in Schizophrenia, delusions, hallucinations, neurocognition issue, social cognition, and social skills [11-13].

\section{Autism spectrum disorders (ASD)}

Studies demonstrated feasibility and potential efficacy of XR for novice ASD drivers' driving-specific executive function (EF) abilities [14,15], Eye gaze [16], social interaction, communication, and repetitive or stereotypic behavior [17].

\section{Attention deficit hyperactivity disorder (ADHD)}

Reliability of attention assessment can be enhanced using XR through better control of the perceptual environment, more consistent stimulus presentation, and by more precise and accurate scoring. Virtual environments (VE) may also lead to validity improvement via the quantification of more discrete behavioral responses, allowing for the identification of more specific cognitive domains. XR could be useful to help testing the attention in situations that are more ecologically valid [18]. Recent studies indicate that the blue-yellow color discrimination is impaired in ADHD individuals. The XR proved to be a user-friendly tool capable to detect and quantify the influence of color on the performance of people executing tasks that require attention and showed to be attractive for people with ADHD [19]. ADHD children are vulnerable to a time on task effect on performances which could explain part of their difficulties. XR-based methods are reliable to test ADHD children ability to sustain performances over time [20].

\section{Mood disorder}

There have also been two studies of (non-immersive) VRtype tasks assessing spatial navigation memory in patients with depression. [10,21]. In an eight days training program, patients exercised the learning and purchasing of shopping list products in a virtual supermarket using either the novel training device or a corresponding desktop application. In a pre-post-design, effects of the two training conditions were compared regarding several outcome measures. Altogether, results did not prove a benefit of the more naturalistic training setting regarding different training performances (recognition, performance speed, and spatial orientation), self-perceived daily cognitive impairments, a real-life shopping task as well as various neuropsychological capabilities [22]. Present evidence suggests that the use of virtual reality has great advantages in evaluating visuospatial navigation and memory for the diagnosis of psychiatric or other neurological disorders concluded that allocentric and egocentric navigation deficits are detectable in patients with schizophrenia and bipolar disorder using VR [23].

\section{Anxiety disorders}

The focus of the treatment studies has typically been on specific phobias or social anxiety or post-traumatic stress disorder, with many fewer investigations for obsessive-compulsive disorder (OCD), which is surprising given that treatment often requires change in fears about external stimuli, and generalized anxiety [10].

\section{Conclusion}

Our point of view is consistent with other authors, who believe in the added value of XR-based technologies including VR, $\mathrm{MR}$ and $\mathrm{AR}$ in treatment of psychiatric disorders and cognitive rehabilitation, compared to the traditional approaches. In the other words, XR make us able to try things that are not easily possible to try in the real world.

\section{References}

1. Moghaddasi H, Sajjadi S, Tabatabaei SM (2016) Mobile Health: An efficient service to promote self-care among people introducing selfcare. J Health Med Inform 7: 223.

2. Chuah S (2018) Why and who will adopt extended reality technology? Literature review, synthesis, and future research agenda, working paper, University Sains Malaysia, School of Management, Department of Operation Management, Malaysia.

3. W. Barfield (2016) Fundamentals of Wearable Computers and Augmented Reality. In: ( $\left.2^{\text {nd }} e d n\right)$, USA.

4. Ro YK, Brem A, Rauschnabel PA (2018) Augmented reality smart glasses: definition, concepts and impact on firm value creation. In: Jung $\mathrm{T}$ and Tom Dieck MC (Eds.), Augmented Reality and Virtual RealityEmpowering Human, Place and Business, USA, pp. 169-181.

5. Suh A, Prophet J (2018) The state of immersive technology research: a literature analysis. Computers in Human Behavior. 86: 77-90.

6. Rauschnabel PA, Rossmann, Andtom MC (2017) An adoption framework for mobile augmented reality games: the case of Pokémon Go. Computers in Human Behavior 76: 276-286.

7. Donnell $\mathrm{O}(2018)$ Driving immersive experiences in virtual and augmented reality, USA.

8. Pan A, Hamilton (2018) Why and how to use virtual reality to study human social interaction: The challenges of exploring a new research landscape. British Journal of Psychology 109(3): 395-417.

9. Pedro G, Jorge O, Carla C, Diogo M, Paulo L, et al. (2017) Cognitive training on stroke patients via virtual reality-based serious games. Disability and Rehabilitation 39(4): 385-388.

10. Freeman, Reeve, Robinson (2017) A Virtual reality in the assessment, understanding, and treatment of mental health disorders. Psychological Medicine 47(14): 2393-2400. 
11. Rus Calafell, Maldonado, Ribas (2014) A virtual reality-integrated program for improving social skills in patients with schizophrenia: a pilot study. J Behav Ther \& Exp Psychiatry 45(1): 81-89.

12. Marques A, Cristina Q, Nuno R (2008) Virtual reality and neuropsychology: a cognitive rehabilitation approach for people with psychiatric disabilities, UK.

13. Wim V(2014) Review and update on virtual reality assessment and treatment in psychosis, Schizophrenia Bulletin 40(6): 1194-1197.

14. Cox DJ, Brown T, Ross (2017) Can youth with autism spectrum disorder use virtual reality driving simulation training to evaluate and improve driving performance? an exploratory study. J Autism Dev Disord 47(8): 2544-2555.

15. Wang M, Reid D (2011) Virtual reality in pediatric neurorehabilitation: attention deficit hyperactivity disorder, autism and cerebral plasy. Neuroepidemiology 36(1): 2-8.

16. Joshua Wade (2015) A virtual reality driving environment for training safe gaze patterns: application in individuals with ASD. Uahci Lncs 9177: 689-697.

17. Bellani M, Fornasari, Chittaro, Brambilla (2011) Virtual reality in autism: state of the art. Epidemiology and Psychiatric Sciences 20(3): 235-238.
18. Parsons TD, Bowerly T, Buckwalter JG, Rizzo AA (2007) A controlled clinical comparison of attention performance in children with adhd in a virtual reality classroom compared to standard neuropsychological methods. Child Neuropsychology 13(4): 363-381.

19. Silva AP, Frere AF (2011) Virtual environment to quantify the influence of colour stimuli on the performance of tasks requiring attention. Biomedical Engineering Online 10: 74.

20. Stephanie B, Lallemand S (2012) Impact of time on task on ADHD patient's performances in a virtual classroom. European Journal of Paediatric Neurology 16(5): 514-521.

21. Hannah C, Traynor V (2015) Computerized and virtual reality cognitive training for individuals at high risk of cognitive decline: systematic review of the literature. American Journal of Geriatric Psychiatry 23(4): 335-359.

22. Lorenz Dehn, Leona Kater (2018) Training in a comprehensive everydaylike virtual reality environment compared to computerized cognitive training for patients with depression. Computers in Human Behavior, pp: 40-52.

23. Mohammadi, Hesami, Kargar, Shams (2018) Detecting allocentric and egocentric navigation deficits in patients with schizophrenia and bipolar disorder using virtual reality. Neuropsychological Rehabilitation 28(3): 398-415. 